\title{
Is fetal-type posterior cerebral artery a risk factor for intracranial aneurysm as analyzed by multislice CT angiography?
}

\author{
ZHEN HE and YEDA WAN \\ The First Department of Radiology, Tianjin Hospital, Tianjin 300202, P.R. China
}

Received April 26, 2017; Accepted August 10, 2017

DOI: $10.3892 /$ etm.2017.5504

\begin{abstract}
Fetal-type posterior cerebral artery (FTP) is a common anatomic variation that is closely associated with intracranial aneurysm. In the present study, multislice computed tomography angiography (CTA) was performed to assess whether FTP is a risk factor for intracranial aneurysm. CTA data of 364 consecutive cases of patients who were suspected with cerebrovascular disease or intracranial aneurysm of intracranial artery from 2013 to 2016 were reviewed and the incidence rates of FTP, other variations of the circle of Willis, intracranial aneurysm and FTP with intracranial aneurysm were evaluated. The $\chi^{2}$ test was used to assess the influence of FTP and gender on the incidence rates of other variations of the circle of Willis, intracranial aneurysm and internal carotid artery-posterior communicating artery (ICA-PComA) aneurysm. Binary logistic regression analysis was performed to assess the associations of FTP and gender with intracranial aneurysm and ICA-PComA aneurysm. Compared with non-FTP patients, FTP cases exhibited significantly higher rates of other variations of the circle of Willis $\left(\chi^{2}=80.173, \mathrm{P}<0.001\right)$ and ICA-PComA aneurysm $\left(\chi^{2}=4.437\right.$, $\mathrm{P}=0.035)$. Among patients with FTP and bilateral FTP, more female than male patients with intracranial aneurysm were identified. However, among all patients with intracranial aneurysm, no statistically significant differences in the prevalence of $\operatorname{FTP}\left(\chi^{2}=2.577, \mathrm{P}=0.108\right)$ and bilateral FTP $\left(\chi^{2}=2.199, \mathrm{P}=0.159\right)$ between males and females were identified. Binary logistic regression analysis revealed that FTP and gender were risk factors for intracranial aneurysm and ICA-PComA aneurysm. A moderate association between FTP and ICA-PComA aneurysm $(\mathrm{OR}=2.762)$ were identified, although there was a weak association between FTP and intracranial aneurysm [odds ratio $(\mathrm{OR})=1.365]$. Furthermore, a strong association was identified between gender and intracranial aneurysm $(\mathrm{OR}=0.328)$, and a
\end{abstract}

Correspondence to: Dr Yeda Wan, The First Department of Radiology, Tianjin Hospital, 406 Jiefang South Road, Hexi, Tianjin 300202, P.R. China

E-mail: hzok@163.com

Key words: fetal-type posterior cerebral artery, intracranial aneurysm, multislice computed tomography angiography, gender, internal carotid artery-posterior communicating artery aneurysm moderate association existed between gender and ICA-PComA aneurysm $(\mathrm{OR}=0.357)$. In conclusion, female gender is an independent risk factor for intracranial aneurysm, and FTP and female gender are independent risk factors for ICA-PComA aneurysm.

\section{Introduction}

Fetal-type posterior cerebral artery (FTP) is a common anatomic variation observed in the circle of Willis, and defined as a posterior cerebral artery that originates from the internal carotid artery (ICA) with or without a small connection with the basilar artery (BA) (1). In this condition, instead of the $\mathrm{BA}$, the ICA supplies blood to the posterior cerebral artery (PCA) (2).

ICA is formed by extending from the paired dorsal aorta towards the cephalic side on embryonic days 28-30 (4-5.7 mm) (3). In the embryonic stage of 5-8 mm, pairs of longitudinal nerve arteries appear along the turbid brain and merge to form basilar arteries. The ICA and the caudal part of the anastomosed branch of the nerve artery form the PCA (4). In the stage of $40 \mathrm{~mm}$ (8 weeks), the PCA appears as an extension of the posterior communicating artery (PComA) (5). The vertebra-basilar system is then formed and contributes to the blood supply of the PCA through the P1 segment. During this period, the components of the circle of Willis have the same diameter (2). During development, the diameter of the PCA-P1 segment gradually increases, while that of the PComA gradually decreases. At the time of birth, the PCA-P1 segment diameter is greater than that of the PComA, which represents the most common type known as the adult PCA (5). Stagnation in the process of embryonic development results in the following two cases. If the P1 segment diameter is similar to that of the PComA, the result is intermediate-type PCA. In the present study, $2.75 \%$ of the subjects had intermediate-type PCA. However, absence of the P1 segment or the diameter of the P1 segment being less than that of the PComA is known as FTP. Absence of the P1 segment reflects full-type FTP. If the diameter of the P1 segment is less than that of the PComA, it is defined as partial-type FTP (6). In the present study, 117 strips of FTP in 91 patients were found, including 40 strips of full-type FTP and 77 strips of partial-type FTP.

Several studies have described FTP in great detail, including its incidence, the association between FTP and occipital lobe infarction, life-threatening headache and white 
matter degeneration $(7,8)$. FTP has also been reported to be associated with the occurrence of PComA aneurysm (9). Thus, it is worthwhile to assess whether FTP is a risk factor for intracranial aneurysm. To the best of our knowledge, the characteristics of intracranial aneurysm, including its incidence, location, association with other variations of the circle of Willis, subarachnoid hemorrhage, aneurysmal morphology (the presence or absence of daughter sac) and size (diameter of the aneurysm neck and aneurysm height), have not been systematically analyzed. Computed tomography angiography (CTA) is a novel and effective examination method for the display of intracranial vessels with the use of three-dimensional reconstruction technology. Due to its great advantage for the detection and diagnosis of intracranial aneurysm over other techniques, it is a preferred choice for diagnosing this condition. In the present study, CTA data of the intracranial artery of 364 consecutive cases of patients who were suspected with cerebrovascular disease or intracranial aneurysm were reviewed, and a detailed analysis was performed to assess the association between FTP and intracranial aneurysm.

\section{Patients and methods}

Subjects. The CTA data of 364 consecutive Chinese cases of intracranial artery assessed at Tianjin Hospital (Tianjin, China) from January 2013 to January 2016 were reviewed. The cohort comprised 218 male and 146 female patients, with an average age of $61.73 \pm 13.33$ years. All the patients were initially diagnosed with cerebral vascular disease or intracranial aneurysm by a neurologist, and the patients' symptoms included headache, vomiting, aphasia, difficulty swallowing, limb weakness, gait instability and hemiplegia. Patients were suggested to undergo CTA examinations of the intracranial artery. This study was approved by the Ethics Committee of Tianjin Hospital (Tianjin, China). Prior to CTA examination, all patients provided written informed consents, which approved the publication of the images and data in the present study.

Examination methods. A 16-slice spiral CT (GE Lightspeed; GE Healthcare, Little Chalfont, UK) and an LF 9000 high-pressure injector (Libel-Flarsheim Company, Cincinnati, $\mathrm{OH}$, USA) were used in this study. The patients entered the scanner in the supine position with the head first. The scan level was from the third cervical vertebra to the calvaria. Scanning conditions were as follows: Scan thickness, $5 \mathrm{~mm}$; pitch, 1.375:1; tube voltage, $140 \mathrm{kV}$; tube current, 200-250 mA. Iohexol (350 mgI/ml, $50 \mathrm{ml}$; Yangtze River Pharmaceutical Group, Taizhou, China) was administered through either the elbow or dorsal vein at a flow rate of $4 \mathrm{ml} / \mathrm{sec}$. The scanning delay time was $20-25 \mathrm{sec}$. The dosage of the contrast agent was $50 \mathrm{ml}$ in $2013-2014$ and $1.0 \mathrm{ml} / \mathrm{kg}$ x body weight $(\mathrm{kg})$ in 2014-2016.

Image processing. Image reconstruction, including volume rendering and the multiplanar reconstructed image (MPR), were obtained on an AW4.5 workstation (GE Healthcare). The reconstruction thickness was $0.625 \mathrm{~mm}$, the window level was $500 \mathrm{HU}$ and the window width was 1,500 HU.
Table I. Standards for the association evaluation of binary logistic regression analysis.

\begin{tabular}{lcl}
\hline OR (lower odds) & OR (higher odds) & Association degree \\
\hline $0.9-1.0$ & $1.0-1.1$ & None \\
$0.7-0.8$ & $1.2-1.4$ & Low \\
$0.4-0.6$ & $1.5-2.9$ & Moderate \\
$0.1-0.3$ & $3.0-9.0$ & Strong \\
$<0.1$ & $>10.0$ & Very strong \\
\hline
\end{tabular}

OR, odds ratio.

Interpretation of images. When the diameter of the PComA was greater than that of the P1 segment of PCA (PCA-P1), partial-type FTP was assumed, while full-type FTP was identified if the PCA-P1 segment was absent (Figs. 1 and 2).

Intracranial aneurysm was defined as abnormal expansion of the artery with congenital, infectious or traumatic causes. According to their shape, aneurysms were divided into saccular and fusiform types (10). Based on their location, saccular aneurysms were divided into bifurcation and lateral wall subtypes (11). ICA-PComA aneurysm is an aneurysm with the neck located in the ICA-PComA. ICA-PComA aneurysms were divided into 5 types according to the location of the aneurysm neck (12). In the present study, 3 types were considered, including the bifurcation (aneurysm neck occupying both ICA and PCA), ICA (aneurysm neck located mainly in the ICA) and PComA (aneurysm neck mainly located in the PComA) types.

Observation. CTA images of the intracranial artery were reviewed to further define FTP occurrence, location (side), type, potential combination with other variations of the circle of Willis (13) and potential combination with intracranial aneurysm.

Regarding aneurysms, the type (fusiform or saccular, bifurcation or lateral wall), location, presence or absence of daughter sac, presence or absence of subarachnoid hemorrhage and potential combination with FTP or other variations of the circle of Willis were assessed. ICA-PComA aneurysms were then classified. The neck of the aneurysm was displayed on an MPR image and the neck diameter and height of the aneurysm were measured.

Statistical analysis. All statistical analyses were performed with SPSS 21.0 software (IBM Corp., Armonk, NY, USA). The incidence of FTP, intracranial aneurysm and FTP with intracranial aneurysm was calculated. The chi-square test was used to assess the influence of FTP and gender on the above items, as well as on aneurysm daughter sac and subarachnoid hemorrhage. The chi-square correction test was performed with a total sample size of $>40$ and a minimum theoretical frequency between 1 and 5. Two independent sample Student's t-tests was used to compare the aneurysm neck diameter and aneurysm height between the FTP and non-FTP patients. Binary logistic regression analysis was performed to assess whether FTP and gender were risk factors for intracranial aneurysm and ICA-PComA aneurysm and the association was evaluated by the standards shown in Table I. 
Table II. Incidence of FTP, bilateral FTP, intracranial aneurysm and intracranial aneurysm associated with FTP/bilateral FTP and influence of gender.

\begin{tabular}{|c|c|c|c|c|c|c|c|c|c|c|}
\hline \multirow[b]{3}{*}{ Variable } & \multirow{2}{*}{\multicolumn{2}{|c|}{ FTP (N1) }} & \multirow{2}{*}{\multicolumn{2}{|c|}{$\begin{array}{l}\text { Bilateral } \\
\text { FTP (N1) }\end{array}$}} & \multirow{2}{*}{\multicolumn{2}{|c|}{$\begin{array}{c}\text { Intracranial } \\
\text { aneurysm (N1) }\end{array}$}} & \multicolumn{2}{|c|}{$\begin{array}{c}\text { Intracranial } \\
\text { aneurysm (N2) }\end{array}$} & \multicolumn{2}{|c|}{ Intracranial aneurysm (N2) } \\
\hline & & & & & & & With & $\overline{\text { Without }}$ & With bilateral & Without bilateral \\
\hline & Yes $(\%)$ & No & Yes $(\%)$ & No & Yes $(\%)$ & No & $\operatorname{FTP}(\%)$ & FTP & FTP $(\%)$ & FTP \\
\hline \multicolumn{11}{|l|}{ Gender } \\
\hline Male & 48 & 170 & 12 & 206 & 18 & 200 & $4(22.22)$ & 14 & $1(5.56)$ & 17 \\
\hline Female & 43 & 103 & 14 & 132 & 32 & 114 & $12(37.5)$ & 20 & $4(12.5)$ & 28 \\
\hline Total incidence & 25.00 & & 7.14 & & $13.74^{\mathrm{a}}$ & & 4.40 & & 1.37 & \\
\hline$\chi^{2}$ & 2.577 & & 2.199 & & 16.524 & & $0.633^{\mathrm{b}}$ & & $0.087^{\mathrm{b}}$ & \\
\hline P-value & 0.108 & & 0.159 & & $<0.001$ & & 0.426 & & 0.768 & \\
\hline
\end{tabular}

ancidence rate of intracranial aneurysm was $13.74 \%$, including saccular (48 aneurysms in 42 patients, 42/364, 11.54\%), fusiform ( 8 aneurysms in 8 patients, $8 / 364,2.2 \%$ ). ' Influence of gender on intracranial aneurysm associated with FTP and bilateral FTP was analyzed using the correction $\chi^{2}$ test. FTP, fetal type of posterior cerebral artery; N1, all patients subjected to computed tomography angiography (218 male and 146 female patients); N2, all patients with intracranial aneurysm (18 males and 32 females).

Table III. Comparison of other variations of the circle of Willis between FTP and non-FTP patients.

\begin{tabular}{lcc}
\hline Other variations of circle of Willis & FTP $(\mathrm{N})$ & Non-FTP $(\mathrm{N})$ \\
\hline Variations of anterior part of circle of Willis & & 5 \\
ACA-A1 hypoplasia & 10 & 2 \\
ACA-A1 absence & 1 \\
Azygos ACA & 1 \\
Trifurcation of ACA & 0 \\
ACA-A1 fenestration & 1 \\
Common trunk of ACA-A2 & 0 \\
AComA fenestration & 6 \\
AComA absence & 2 \\
Duplication MCA & 2 \\
Early bifurcation of MCA & 1 \\
Variations of posterior part of circle of Willis & 2 \\
PComA absence & 25 \\
Duplication PCA & 4 \\
Hyperplastic anterior choroidal artery & 2 & 1 \\
BA fenestration & 2 & 3 \\
VA fenestration & 0 & 1 \\
Total (N/n) & $45 / 76^{\mathrm{a}}$ & 0
\end{tabular}

a9 patients with 2 other variations of circle of Willis; 5 patients with 3 other variations of Circle of Willis. b8 patients with 2 other variations of circle of Willis, 3 patients with 3 other variations of circle of Willis. FTP, fetal type of posterior cerebral artery; N, number of patients; $\mathrm{n}$, number of other variations of circle of Willis; ACA-A1, A1 segment of anterior cerebral artery; AComA, anterior communicating artery; MCA, middle cerebral artery; PComA, posterior communicating artery; BA, basilar artery; VA, vertebral artery; PCA, posterior cerebral artery.

\section{Results}

Incidence of FTP. The total incidence of FTP and bilateral FTP (Fig. 3) was 25.00 and 7.14\%, respectively (Table II). There was no statistical difference between the total incidence of FTP and bilateral FTP between males and females $\left(\chi^{2}=2.577\right.$, $\mathrm{P}=0.108)$. A total of 117 strips of FTP were identified, including
77 strips of partial-type FTP and 40 strips of full-type FTP. In addition, 10 patients $(2.75 \%$ in total) with intermediate-type PCA were identified.

Other variations of the circle of Willis in FTP and non-FTP patients are presented in Table III. The percentage of other variations of the circle of Willis in patients with and without FTP was 49.45 and $7.69 \%$, respectively (Table IV). There was a 


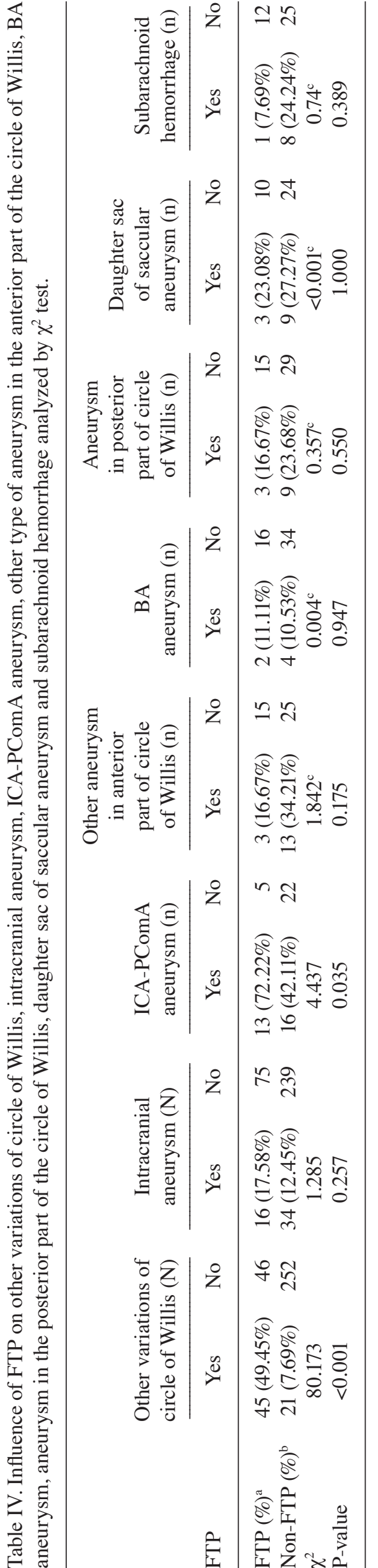

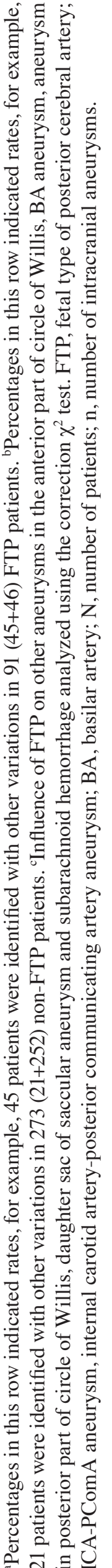

Table V. Variations in circle of Willis associated with intracranial aneurysm in a total of 50 patients with intracranial aneurysms.

\begin{tabular}{lc}
\hline $\begin{array}{l}\text { Variations in circle } \\
\text { of Willis }\end{array}$ & $\begin{array}{c}\text { Patients associated with } \\
\text { intracranial aneurysm (N/\%) }\end{array}$ \\
\hline FTP & $\begin{array}{c}15 \text { (10 of 15 accompanied with other } \\
\text { variations of circle of Willis, 30) } \\
\text { (3 of 7 accompanied with FTP 14) }\end{array}$ \\
ACA-A1 absence & 5 (3 of 7 accompanied with FTP, 10) \\
ACA-A1 hypoplasia & $1(2)$ \\
Azygos ACA & $1(2)$ \\
ACmoA fenestration & $3(6)$ \\
ACmoA absence & $2(4)$ \\
Duplication MCA & 1 (accompanied with FTP, 2) \\
Early bifurcation of & \\
MCA & 6 (3 of 6 accompanied with FTP, 12) \\
PComA & 31 (62) \\
Total &
\end{tabular}

FTP, fetal type of posterior cerebral artery; ACA-A1, A1 segment of anterior cerebral artery; MCA, middle cerebral artery; PComA, posterior communicating artery; $\mathrm{N}$, number of patients.

Table VI. Influence of daughter sac associated with subarachnoid hemorrhage in saccular aneurysms.

\begin{tabular}{lcc}
\hline $\begin{array}{l}\text { Daughter sac of } \\
\text { saccular aneurysm }\end{array}$ & $\begin{array}{c}\text { With } \\
\text { Subarachnoid } \\
\text { hemorrhage (n) }\end{array}$ & $\begin{array}{c}\text { Without } \\
\text { Subarachnoid } \\
\text { hemorrhage (n) }\end{array}$ \\
\hline Yes & 6 & 6 \\
No & 3 & 33 \\
Continuity correction $\chi^{2}$ & 7.704 & \\
P-value & 0.006 & \\
\hline
\end{tabular}

Data were analyzed using the continuity correction $\chi^{2}$ test. $\mathrm{N}$, number of saccular aneurysms.

statistical difference on other variations of the circle of Willis between FTP and non-FTP patients $\left(\chi^{2}=80.173, \mathrm{P}<0.001\right)$.

Incidence of intracranial aneurysms. Within the cohort $(\mathrm{n}=364), 50$ patients $(13.74 \%$; 18 males and 32 females; mean age, $61.66 \pm 14.03$ years) with 56 intracranial aneurysms were identified, including $11.54 \%$ (42/364; 48 aneurysms in 42 patients) saccular aneurysms and $2.20 \%$ (8/364; 8 aneurysms in 8 patients) fusiform aneurysms (Table II). Among them, multiple aneurysms accounted for $1.37 \%(5 / 364 ; 11$ aneurysms in 5 patients). The dimensions of the intracranial saccular aneurysms were $2.93 \pm 1.6 \mathrm{~mm}$ (aneurysm neck diameter) x3.52 $\pm 2.47 \mathrm{~mm}$ (aneurysm height).

Of the intracranial aneurysms, 62\% (31/50) were associated with variations of the circle of Willis, particularly the FTP variation (Table V). Among the 48 saccular aneurysms, 12 were accompanied with a daughter sac and 9 with 
Table VII. Influence of daughter sac on subarachnoid hemorrhage in saccular aneurysms.

\begin{tabular}{lccccccc}
\hline Risk factor & $\mathrm{B}$ & $\mathrm{SE}$ & Wald $\left(\chi^{2}\right)$ & df & P-value & Exp(B) $(\mathrm{OR})$ & $95.0 \% \mathrm{CI}$ for Exp(B) \\
\hline Daughter sac & 2.398 & 0.835 & 8.250 & 1 & 0.004 & 11.000 & $2.142-56.496$
\end{tabular}

Data were analyxed using binary logistic regression analysis. FTP, fetal type of posterior cerebral artery; B, slope coefficient; SE, standard error; df, degrees of freedom; OR, odds ratio; CI, confidence interval; Exp, Exponential.

Table VIII. Comparison of the location of intracranial aneurysms between FTP and non-FTP patients.

\begin{tabular}{lcc}
\hline Location of intracranial aneurysm & Intracranial aneurysm with FTP (N/\%) & Intracranial aneurysm without FTP $(\mathrm{N} / \%)$ \\
\hline Anterior part of circle of Willis & $2^{\mathrm{a}}(12.5)$ & $4^{\mathrm{b}}(11.76)$ \\
ACA & 0 & $1(2.94)$ \\
AComA & 0 & $3(8.82)$ \\
Trifurcation of ICA-ACA-MCA & $11^{\mathrm{c}}(13$ aneurysms, 2 fusiform aneurysms & $13^{\mathrm{d}}(16$ aneurysms $)(38.24)$ \\
ICA-PcomA & among them $)(68.75)$ & $5(1$ fusiform aneurysm $)(14.71)$ \\
MCA & $1(6.25)$ & $1(2.94 \%)$ \\
Posterior part of circle of Willis & $1(6.25)$ & $4(2$ fusiform aneurysms $)(11.76)$ \\
PCA & $2(1$ fusiform aneurysm $)(12.5)$ & $4(2$ fusiform aneurysms $)(11.76)$ \\
BA & 0 & $34(38$ aneurysms $)$ \\
VA & $16(18$ aneurysms $)$ &
\end{tabular}

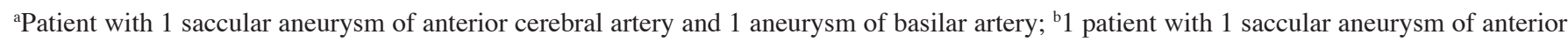
cerebral artery and 1 saccular aneurysm of anterior communicating artery; ${ }^{c} 1$ patient with 3 saccular aneurysms of internal carotid artery-posterior communicating artery; ${ }^{\mathrm{d}} 3$ patients with 6 saccular aneurysms of internal carotid artery-posterior communicating artery ( 2 saccular aneurysms per patient). FTP, fetal type of posterior cerebral artery; ACA, anterior cerebral artery; AComA, anterior communicating artery; ICA, internal carotid artery; PComA, posterior communicating artery; MCA, middle cerebral artery; PCA, posterior cerebral artery; BA, basilar artery; VA, vertebral artery; $\mathrm{N}$, number of patients.

Table IX. Comparison of ICA-PComA aneurysm type between FTP and non-FTP patients.

Type of ICA-PComA aneurysm ICA-PComA aneurysm with FTP $(\mathrm{N} / \mathrm{n})\left(\%^{\mathrm{a}}\right) \quad$ ICA-PComA aneurysm without FTP $(\mathrm{N} / \mathrm{n})\left(\%^{\mathrm{b}}\right)$

\begin{tabular}{lcc}
\hline Bifurcation & $1 / 1(1.10)$ & $0 / 0$ \\
ICA & $8 / 10^{\mathrm{a}}(8.79)$ & $12 / 15^{\mathrm{b}}(4.56)$ \\
PComA & $2 / 2^{\mathrm{a}}(2.20)$ & $1 / 1(0.38)$ \\
Total & $11 / 13(12.09)$ & $13 / 16(4.94)$ \\
\hline
\end{tabular}

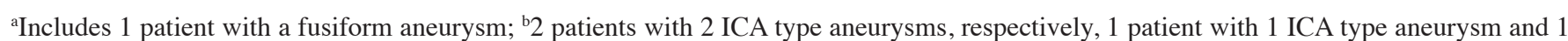
aneurysm of middle cerebral artery. ICA, internal carotid artery; PComA, posterior communicating artery; FTP, fetal type of posterior cerebral artery; N, number of patients; $\mathrm{n}$, number of ICA-PComA aneurysms.; $\%^{\mathrm{a}}$, the percentage of ICA-PComA aneurysm in FTP patients; $\%$, the percentage of ICA-PComA aneurysm in non-FTP patients.

subarachnoid hemorrhage. In addition, 6 saccular aneurysms had an aneurysm daughter sac combined with subarachnoid hemorrhage. The probability of subarachnoid hemorrhage in intracranial saccular aneurysm with daughter sac was significantly higher than that in saccular aneurysm without daughter sac, as analyzed by Continuity Correction $\chi^{2}$ test $\left(\chi^{2}=7.704\right.$, $\mathrm{P}=0.006$; Table VI). In addition, the presence of an aneurysm daughter sac was closely associated with subarachnoid hemorrhage as analyzed by binary logistic regression, with a strong association $(\mathrm{OR}=11.000$; Table VII).

Intracranial aneurysms in FTP. The incidence of intracranial aneurysm with FTP in all patients was $4.40 \%(16 / 364)$ (Table II). The rates of intracranial aneurysm combined with unilateral or bilateral FTP in female patients $(37.5 \%$ for unilateral and $12.5 \%$ for bilateral FTP) were higher compared with 
Table X. Influence of FTP and gender on intracranial aneurysm by binary logistic regression analysis.

\begin{tabular}{lccccccc}
\hline Risk factor & $\mathrm{B}$ & $\mathrm{SE}$ & Wald $\left(\chi^{2}\right)$ & $\mathrm{df}$ & P-value & $\operatorname{Exp}(\mathrm{B})(\mathrm{OR})$ & $95.0 \%$ CI for $\operatorname{Exp}(\mathrm{B})$ \\
\hline FTP & 0.311 & 0.338 & 0.846 & 1 & 0.358 & 1.365 & $0.703-2.649$ \\
Gender & -1.116 & 0.318 & 12.296 & 1 & 0.000 & 0.328 & $0.176-0.611$ \\
\hline
\end{tabular}

FTP, fetal type of posterior cerebral artery; B, slope coefficient; SE, standard error; df, degrees of freedom; OR, odds ratio; CI, confidence interval; Exp, Exponential.

Table XI. Influence of FTP and gender on ICA-PComA aneurysm by binary logistic regression analysis.

\begin{tabular}{lccccccc}
\hline Risk factor & $\mathrm{B}$ & $\mathrm{SE}$ & Wald $\left(\chi^{2}\right)$ & $\mathrm{df}$ & P-value & $\operatorname{Exp}(\mathrm{B})(\mathrm{OR})$ & $95.0 \%$ CI for $\operatorname{Exp}(\mathrm{B})$ \\
\hline FTP & 1.016 & 0.442 & 5.292 & 1 & 0.021 & 2.762 & $1.162-6.563$ \\
Gender & -1.029 & 0.456 & 5.091 & 1 & 0.024 & 0.357 & $0.146-0.874$ \\
\hline
\end{tabular}

FTP, fetal type of posterior cerebral artery; B, slope coefficient; SE, standard error; df, degrees of freedom; OR, odds ratio; CI, confidence interval; Exp, Exponential.

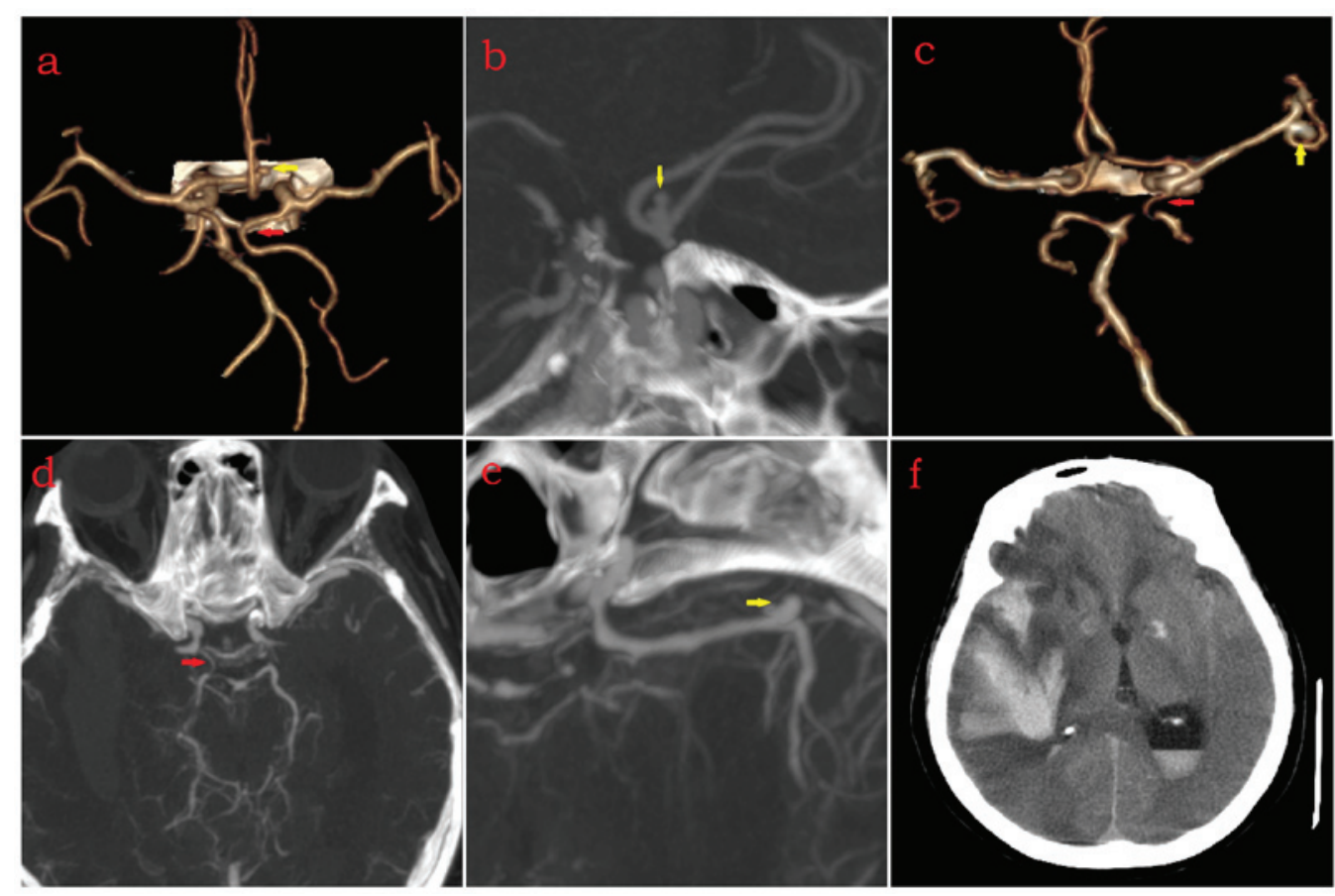

Figure 1. Right full or partial FTP. (a and b) Female (age, 75 years) with right full-type FTP (red arrow), saccular aneurysm with daughter sac located at bifurcation of bilateral ACA-A2 (yellow arrow) and absence of right ACA-A1. (c-f) Female (age, 75 years) with right partial-type FTP (red arrow), saccular aneurysm located at bifurcation of MCA-M1 and MCA-M2 (yellow arrow), absence of left posterior communicating artery, and subarachnoid hemorrhage. ACA-A1, A1 segment of anterior cerebral artery; MCA, middle cerebral artery; M1, M1 segment; FTP, fetal type of posterior cerebral artery.

those in males $(22.22 \%$ for unilateral and $5.56 \%$ for bilateral) (Table II). The incidence of FTP in intracranial aneurysm patients was $32 \%(16 / 50)$, including $10 \%(5 / 50)$ of bilateral FTP cases. The incidence rate of intracranial aneurysm in FTP patients was $17.58 \%$ (16/91), which was slightly higher than that in non-FTP patients with $12.45 \%(34 / 273)$, but the difference was not statistically significant $\left(\chi^{2}=1.285, \mathrm{P}=0.257\right.$; Table IV). Table VIII presents the location of intracranial aneurysm in FTP and non-FTP patients.
Incidence of FTP and ICA-PComA aneurysm. In the present study, 29 ICA-PComA aneurysms (24 patients) were identified. Of the patients with FTP, $12.09 \%$ (11/91) presented with ICA-PComA aneurysm and 2.20\% (2/91) of cases occurred in the initial part of PComA (Table IX), and were predominantly identified in females. Table IX displayed the ICA-PComA aneurysm type in FTP and non-FTP patients. The ICA type was predominant type in FTP or non-FTP patients. With the exception of ICA-PComA aneurysm, there was no statistical 


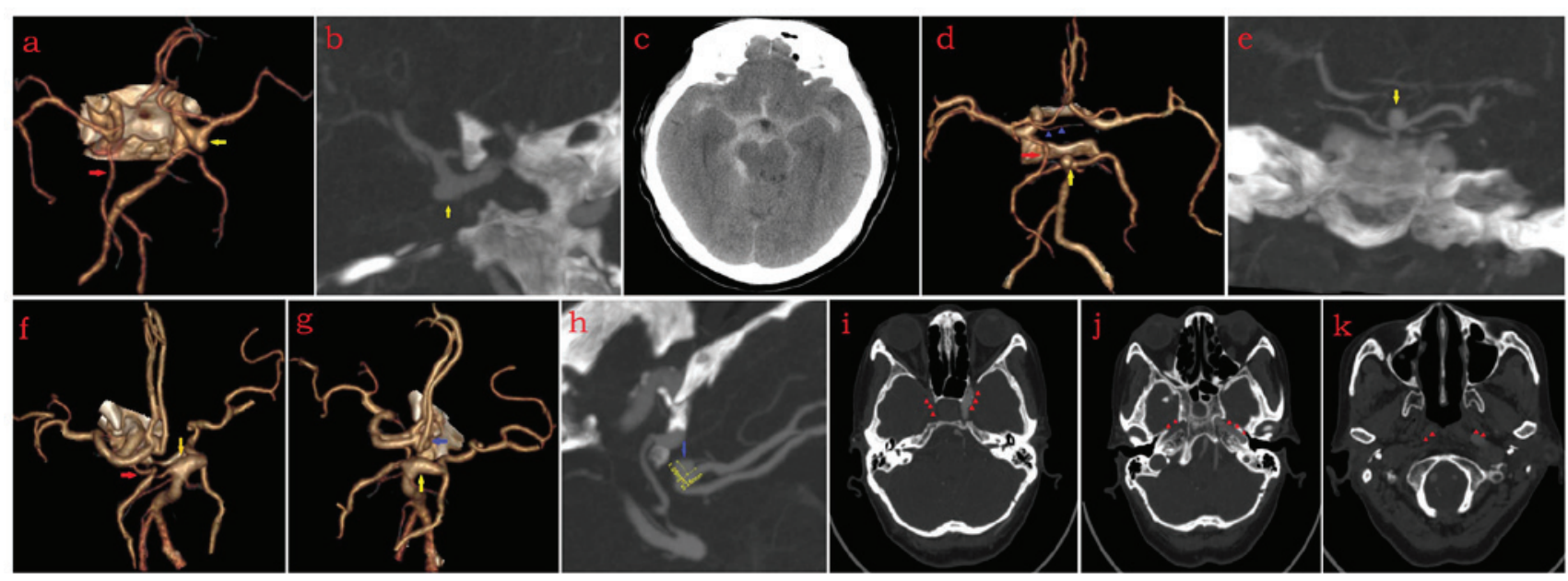

Figure 2. Left full or partial FTP. (a-c) Male (age, 64 years) with left full FTP (red arrow), saccular ICA-PComA aneurysm (ICA type, yellow arrow), left ACA-A1 hyposplasia, absence of anterior communicating artery, absence of right PComA, and subarachnoid hemorrhage. (d and e) Female (age, 75 years) with left partial-type FTP (red arrow), saccular aneurysm located at bottom of BA (yellow arrow), left heubner recurrent artery (blue arrows head) and absence of right PComA. (f-k) Female (age, 70 years) with left partial-type FTP (red arrow), fusiform aneurysm located at transition between BA and right posterior cerebral artery (yellow arrow), saccular aneurysm located at bifurcation of bilateral ACA-A2 (blue arrow) (neck diameter of aneurysm, $5.16 \mathrm{~mm}$; height of aneurysm, $3.89 \mathrm{~mm}$ ), absence of right ACA-A1 and absence of right ICA (blue arrow heads). ICA, internal carotid artery; ACA-A1, A1 segment of anterior cerebral artery; PComA, posterior communicating artery; BA, basilar artery; ICA, internal carotid artery; FTP, fetal type of posterior cerebral artery.

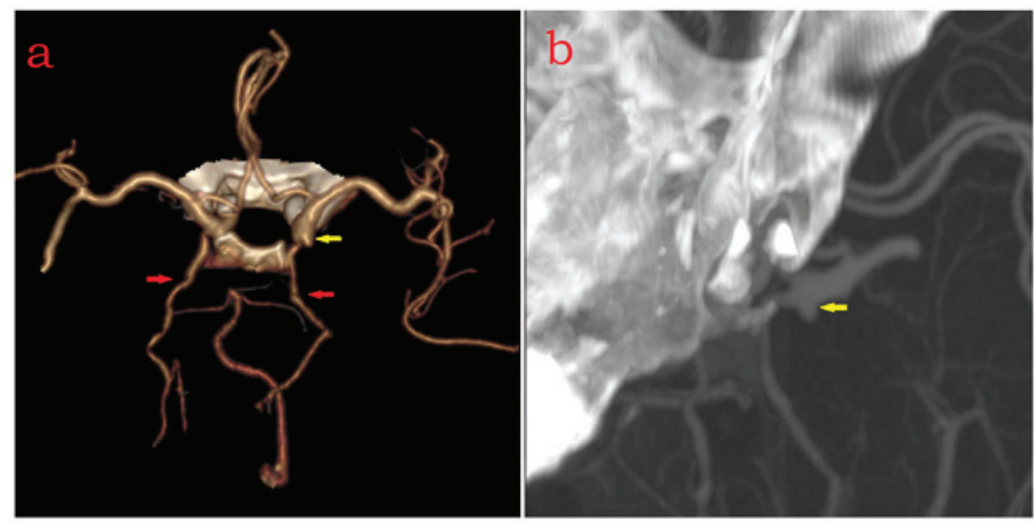

Figure 3. Bilateral FTP and intracranial aneurysm. (a) Overview of bilateral FTP and intracranial aneurysm. (b) ICA-PComA aneurysm in the same patient presented in detail. (a and b) Female (age, 70 years) with right partial-type FTP (red arrow), left full-type FTP (red arrow), right saccular ICA-PComA aneurysm (ICA type, yellow arrow) and absence of left PComA. ICA, internal carotid artery; PComA, posterior communicating artery; FTP, fetal type of posterior cerebral artery.

difference regarding intracranial aneurysms at other positions between FTP and no-FTP patients (Table IV).

Influences of FTP and gender on intracranial aneurysm and ICA-PComA aneurysm. Table II indicates the incidence of FTP, bilateral FTP, intracranial aneurysm, intracranial aneurysm with FTP and intracranial aneurysm with bilateral FTP between females and males by the $\chi^{2}$ test. A statistically significant difference in the incidence of intracranial aneurysm between females and males was identified $\left(\chi^{2}=16.524\right.$, $\mathrm{P}<0.001)$. More females than males had intracranial aneurysm with FTP and bilateral FTP; however, the difference was not significant. No statistically significant differences in the incidence of any of the other conditions mentioned above were noted between females and males.

Table IV displays the influence of FTP on other variations of the circle of Willis, intracranial aneurysm, ICA-PComA aneurysm, other aneurysms in the anterior part of the circle of Willis, BA aneurysm, aneurysm in the posterior part of the circle of Willis, daughter sac of saccular aneurysm and subarachnoid hemorrhage as analyzed by the $\chi^{2}$ test. Statistically significant differences in the incidence of other variations of the circle of Willis $\left(\chi^{2}=80.173, \mathrm{P}<0.001\right)$ and ICA-PComA aneurysm $\left(\chi^{2}=4.437, \mathrm{P}=0.035\right)$ were identified between FTP and non-FTP patients (Table IV). No statistically significant differences in the incidence of any of the other conditions mentioned above were noted between FTP and non-FTP patients.

Table X presents the association of FTP and gender with intracranial aneurysm. A weak association was identified between FTP and intracranial aneurysm $(\mathrm{OR}=1.365)$, while there was a stronger association between gender and intracranial aneurysm $(\mathrm{OR}=0.328)$.

Table XI displays the association of FTP and gender with ICA-PComA aneurysm. A moderate association was identified between FTP and ICA-PComA aneurysm (OR=2.762). In 
addition, a moderate association was present between gender and ICA-PComA aneurysm $(\mathrm{OR}=0.357)$.

\section{Discussion}

FTP is a posterior cyclic variation of the circle of Willis. Blood supply of the PCA on the FTP side is exclusively from the ipsilateral ICA, or from both the ipsilateral ICA and the BA, but predominantly from the ICA. Under normal circumstances, intracranial blood supply on both sides simultaneously relies on the cervical and vertebral basilar system, and the cerebral blood flow pressure remains similar between both sides. In the case of FTP, the blood flow of the ICA and vertebral basilar system is unbalanced, leading to a series of hemodynamic changes in circle of Willis components (14). First, blood flow is increased in the ICA-PComA and the blood pressure is enhanced, leading to increased impact on the vessel wall (15). Furthermore, the membrane lacks the muscle layer in the blood vessel wall of the arterial bifurcation and the blood vessel wall appears to be thinning (16).

In addition, the present study identified some other variations of the circle of Willis in FTP and non-FTP patients. The percentage of other variations of the circle of Willis in patients with and without FTP was 49.45 and $7.69 \%$, respectively. The former was identified to be significantly higher compared with the latter. In theory, the hemodynamic changes of the circle of Willis would be more complex if FTP was combined with other variations (17). Previous studies have reported that anatomical variations of the circle of Willis, including persistent trigeminal artery, arterial window and anterior cerebral artery (ACA) -A1 dysplasia or absence, are associated with the occurrence of intracranial aneurysm $(18,19)$. In fact, the present study also indicated that the incidence of intracranial aneurysm in FTP with other variations of the circle of Willis was higher than that in non-FTP patients; however, there was no significant difference between them. In addition, some variations in the circle of Willis were demonstrated to be associated with intracranial aneurysm in the present study. Among them, FTP was the most common variation associated with intracranial aneurysm. The incidence of FTP in intracranial aneurysm patients was $30 \%$, including $10 \%$ for bilateral FTP cases, which was in line with the results of a previous study (20).

In 50 patients with intracranial aneurysm, there were 18 males and 32 females. There was significant difference between females and males who had intracranial aneurysms. Of the 48 intracranial saccular aneurysms identified in the present study, 12 had a daughter sac, 9 occurred with subarachnoid hemorrhage and 6 simultaneously occurred with both aneurysm daughter sac and subarachnoid hemorrhage. Analysis by Continuity Correction $\chi^{2}$ test revealed that saccular aneurysms with daughter sacs demonstrated a higher chance of subarachnoid hemorrhage, which was consistent with previous study (21). Furthermore, 3 saccular aneurysms were present with the daughter sac and FTP, and 1 saccular aneurysm was indicated with subarachnoid hemorrhage and FTP. However, no association between FTP and subarachnoid hemorrhage was identified in the present study. Results demonstrated the location of intracranial aneurysm, including the ACA, AComA, ICA-PComA, MCA, PCA and BA between
FTP and non-FTP patients. Regardless of patients with FTP and patients without FTP, ICA-PComA aneurysm accounted for the largest proportion.

As mentioned above, there was no statistically significant difference between FTP and non-FTP patients regarding the incidence of intracranial aneurysm. However, a statistical difference was identified between FTP and non-FTP with ICA-PComA aneurysms. No significant differences were determined between FTP and non-FTP in intracranial aneurysms located elsewhere. In the present study, the ICA-PComA aneurysms were divided into 3 types, including the bifurcation type (aneurysm neck occupying both ICA and PCA), the ICA type and the PComA type. The ICA type was the predominant type in FTP and non-FTP patients. These results corroborated with the findings of Zada et al (22). Of note, ICA-PcomA aneurysm require distinguishing from the PComA funnel due to differences in treatment (12); the PComA funnel is a variation which does not require surgical treatment.

Binary logistic regression analysis revealed that gender was a risk factor for intracranial aneurysm and ICA-PComA aneurysm. A strong association was identified between gender and intracranial aneurysm $(\mathrm{OR}=0.328)$, and a moderate association between gender and ICA-PComA aneurysm (OR=0.357). Among patients with unilateral and bilateral FTP, more female than male patients with intracranial aneurysm were identified. This result was in accordance with that of a previous study, which proved that the prevalence of unruptured intracranial aneurysms in women was higher than that in men (23). The significant difference in the prevalence between males and females may be due to estrogen levels (24), which are also easily influenced by age, and the interplay among these factors deserves further research.

In conclusion, the present study indicated that female is an independent risk factor for intracranial aneurysm, and FTP and female are independent risk factors for ICA-PcomA aneurysm. It is known that age, gender, smoking, alcohol consumption, hypertension, coronary heart disease and diabetes are risk factors for intracranial aneurysm $(25,26)$. Therefore, clinicians should pay sufficient attention to female patients with FTP, and a comprehensive follow-up program combined with risk factors of other aneurysms should be designed for the early prevention and treatment of intracranial aneurysm.

\section{References}

1. Arjal RK, Zhu T and Zhou Y: The study of fetal-type posterior cerebral circulation on multislice CT angiography and its influence on cerebral ischemic strokes. Clin Imaging 38: 221-225, 2014.

2. Lv X, Li Y, Yang X, Jiang C and Wu Z: Potential proneness of fetal-type posterior cerebral artery to vascular insufficiency in parent vessel occlusion of distal posterior cerebral artery aneurysms. J Neurosurg 117: 284-287, 2012.

3. Alexandre AM, Visconti E, Schiarelli C, Frassanito P and Pedicelli A: Bilateral internal carotid artery segmental agenesis: Embryology, common collateral pathways, clinical presentation and clinical importance of a rare condition. World Neurosurg 95: 620.e9-620.e15, 2016.

4. Xu J, Xu L, Wu Z, Chen X, Yu J and Zhang J: Fetal-type posterior cerebral artery: The pitfall of parent artery occlusion for ruptured $\mathrm{P}_{2}$ segment and distal aneurysms. J Neurosurg 123: 906-914, 2015

5. Hu T and Wang D: Association between anatomical variations of the posterior communicating artery and the presence of aneurysms. Neurol Res: 1-7, 2016 (Epub ahead of print). 
6. Tocco P, Fenzi F, Cerini R and Monaco S: Adult-onset migraine-related ophthalmoplegia and omolateral fetal-type posterior cerebral artery. BMJ Case Rep 2011: pii: bcr1020114930, 2011.

7. Diogo MC, Fragata I, Dias SP, Nunes J, Pamplona J and Reis J: Low prevalence of fetal-type posterior cerebral artery in patients with basilar tip aneurysms. J Neurointerv Surg 9 : 698-701, 2017.

8. Kolukisa M, Gursoy AE, Kocaman G, Dürüyen H, Toprak H and Asil T: Carotid endarterectomy in a patient with posterior cerebral artery infarction: Influence of Fetal Type PCA on atypical clinical course. Case Rep Neurol Med 2015: 191202, 2015.

9. Lv N, Feng Z, Wang C, Cao W, Fang Y, Karmonik C, Liu J and Huang Q: Morphological risk factors for rupture of smal $(<7 \mathrm{~mm})$ posterior communicating artery aneurysms. World Neurosurg 87: 311-315, 2016.

10. Cron DC, Coleman DM, Sheetz KH, Englesbe MJ and Waits SA Aneurysms in abdominal organ transplant recipients. J Vasc Surg 59: 594-598, 2014

11. Huang DZ, Jiang B, He W, Wang YH and Wang ZG: Risk factors for the recurrence of an intracranial saccular aneurysm following endovascular treatment. Oncotarget 8: 33676-33682, 2017.

12. González-Darder JM, Quilis-Quesada V, Talamantes-Escribá F, Botella-Maciá L and Verdú-López F: Microsurgical relations between internal carotid artery-posterior communicating artery (ICA-PComA) segment aneurysms and skull base: An anatomoclinical study. J Neurol Surg B Skull Base 73: 337-341, 2012.

13. Kim KM, Kang HS, Lee WJ, Cho YD, Kim JE and Han MH: Clinical significance of the circle of Willis in intracranial atherosclerotic stenosis. J Neurointerv Surg 8: 251-255, 2016.

14. Lochner P, Golaszewski S, Caleri F, Ladurner G, Tezzon F, Zuccoli G and Nardone R: Posterior circulation ischemia in patients with fetal-type circle of Willis and hypoplastic vertebrobasilar system. Neurol Sci 32: 1143-1146, 2011.

15. Xu J, Yu Y, Wu X, Wu Y, Jiang C, Wang S, Huang Q and Liu J: Morphological and hemodynamic analysis of mirror posterior communicating artery aneurysms. PLoS One 8: e55413, 2013.

16. Lin W, Ma X, Deng D and Li Y: Hemodynamics in the circle of Willis with internal carotid artery stenosis under cervical rotatory manipulation: A Finite element analysis. Med Sci Monit 21: 1820-1826, 2015

17. Law-Ye B, Geerts B, Galanaud D, Dormont D and Pyatigorskaya N: Pseudo-asymmetry of cerebral blood flow in arterial spin labeling caused by unilateral fetal-type circle of Willis: Technical limitation or a way to better understanding physiological variations of cerebral perfusion and improving arterial spin labeling acquisition? J Cereb Blood Flow Metab 36: 1641-1643, 2016.
18. Patel MA, Caplan JM, Yang W, Colby GP, Coon AL, Tamargo RJ and Huang J: Arterial fenestrations and their association with cerebral aneurysms. J Clin Neurosci 21: 2184-2188, 2014

19. Orakdöğen M, Emon ST, Somay H, Engin T, Is M and Hakan T: Vascular variations associated with intracranial aneurysms. Turk Neurosurg 27: 853-862, 2017.

20. Ilbay K, Ismailoglu $\mathrm{O}$ and Albayrak BS: Co-existence of bilateral fetal type posterior cerebral artery and the bilateral giant internal carotid artery aneurysms in an ataxic patient. Eur J Radiol 81: 1388-1389, 2012

21. Hao M, Ma J, Huang QJ, He SX, Liang Z and Wang CB: Morphological parameters of digital subtraction angiography 2D image in rupture risk profile of small intracrania Aneurysms: A Pilot Study.J Neurol Surg ACentEurNeurosurg 77: 25-30, 2016

22. Zada G, Breault J, Liu CY, Khalessi AA, Larsen DW, Teitelbaum GP and Giannotta SL: Internal carotid artery aneurysms occurring at the origin of fetal variant posterior cerebral arteries: surgical and endovascular experience. Neurosurgery 63 (1 Suppl 1): ONS55-ONS62, 2008

23. Harada K, Fukuyama K, Shirouzu T, Ichinose M, Fujimura H, KakumotoK and Yamanaga Y: Prevalence of unruptured intracranial aneurysms in healthy asymptomatic Japanese adults: Differences in gender and age. Acta Neurochir (Wien) 155: 2037-2043, 2013.

24. Tada Y, Wada K, Shimada K, Makino H, Liang EI, Murakami S, Kudo M, Shikata F, Pena Silva RA, Kitazato KT, et al: Estrogen protects against intracranial aneurysm rupture in ovariectomized mice. Hypertension 63: 1339-1344, 2014.

25. Brinjikji W, Zhu YQ, Lanzino G, Cloft HJ, Murad MH, Wang Z and Kallmes DF: Risk factors for growth of intracranial aneurysms: A systematic review and meta-analysis. AJNR Am J Neuroradiol 37: 615-620, 2016.

26. Kang HG, Kim BJ, Lee J, Kim MJ, Kang DW, Kim JS and Kwon SU: Risk factors associated with the presence of unruptured intracranial aneurysms. Stroke 46: 3093-3098, 2015.

This work is licensed under a Creative Commons Attribution-NonCommercial-NoDerivatives 4.0 International (CC BY-NC-ND 4.0) License. 